\title{
A new strategy for cryptic telomeric translocation screening in patients with idiopathic mental retardation
}

\author{
Saeed R Ghaffari, Elizabeth Boyd, J L Tolmie, Yanick J Crow, Alison H Trainer, \\ J Michael Connor
}

\begin{abstract}
Cryptic unbalanced chromosome rearrangements in the telomeric bands of human chromosomes constitute a significant cause of "idiopathic" mental retardation. Here, we have described a new strategy based upon comparative genomic hybridisation (CGH) to screen for these abnormalities. A modified CGH analysis showed three unbalanced cryptic rearrangements in five patients from three families. These chromosome abnormalities and their balanced forms in the relatives were then confirmed by fluorescence in situ hybridisation (FISH). This study describes a new approach to the diagnosis of cryptic translocations between the $G$ band negative ends of chromosomes and confirms the significant contribution of cryptic telomeric rearrangements to idiopathic mental retardation.

$(\Im$ Med Genet 1998;35:225-233)
\end{abstract}

Keywords: mental retardation; comparative genomic hybridisation; telomere

Molecular cytogenetic and DNA methods are increasingly used to diagnose cytogenetically invisible translocations involving the $G$ band negative ends of chromosomes in patients who have a normal $G$ banded karyotype..$^{1-5}$ When clinical features suggestive of a known chromosome syndrome are present, it is straightforward to investigate the possibility of a translocation with specific DNA probes by using fluorescence in situ hybridisation (FISH) or other molecular methods. ${ }^{6-21}$ However, in routine clinical practice, the majority of people with unexplained mental handicap do not have clinical signs that suggest one particular chromosome abnormality, although in certain cases there may be a strong clinical suspicion of an underlying chromosome imbalance. Since cryptic rearrangements that cause mental handicap tend to concentrate in gene rich subtelomeric regions, ${ }^{22}$ a screening strategy was recently described which uses a combination of molecular cytogenetic and DNA techniques to screen for subtelomeric chromosomal abnormalities. ${ }^{5}$ This approach constitutes a very powerful diagnostic tool but several experiments have to be done on each case to confirm or exclude a telomeric rearrangement. Moreover, only chromosomal regions that are specified by the DNA probes used will be screened. Therefore, it would be desirable to develop an alternative method that can screen the whole genome for cryptic chromosome imbalance in a single experiment.

Comparative genomic hybridisation (CGH) has the potential to provide genome wide screening for cryptic unbalanced aberrations. CGH is a modified FISH technique in which differentially labelled test and reference DNA is cohybridised on normal "target" metaphase chromosomes to assay copy number differences. ${ }^{23}{ }^{24}$ Since its development, CGH has been applied mostly as a research tool in the field of cancer cytogenetics, but it has also been used as an adjunct to traditional cytogenetic banding analysis. ${ }^{25-27}$ The smallest possible chromosome abnormality that $\mathrm{CGH}$ will detect has not yet been defined and, importantly, telomeric regions have previously been excluded from $\mathrm{CGH}$ analysis because the absolute green and red fluorescence intensities gradually decrease at the telomeres and unreliable ratio changes may appear as the fluorescence intensities approach background fluorescence. ${ }^{25} 26$

Here, we show that if certain criteria are met, CGH can be adapted successfully to detect cryptic terminal translocations. Three families in which cytogenetic analysis by standard G banding (400-500 band level) and higher resolution studies had failed to detect an abnormality were studied by modified CGH. Subtelomeric imbalance was identified in each family and the findings were confirmed by targeted FISH.

\section{Methods}

PATIENT SELECTION

Three families were carefully chosen for study. In each, two affected members with an apparently normal karyotype and a similar phenotype were related through at least one healthy first degree relative. These features are highly suggestive of the segregation of a cryptic chromosomal translocation.

\section{METAPHASE SPREADS}

Metaphase spreads were prepared from phytohaemagglutinin (PHA) stimulated, methotrexate synchronised peripheral blood lymphocytes from patients (for FISH) or healthy males (for $\mathrm{CGH}$ ) using standard procedures of hypotonic treatment and methanol/acetic acid fixation $(3: 1, v / v)$.

GENOMIC DNA PROBES AND LABELLING PROCEDURES FOR CGH EXPERIMENTS

Test and control DNA was extracted by proteinase $\mathrm{K}$ and $\mathrm{RNase}$ digestions. Control genomic 
DNA was prepared from the blood of healthy males $(46, \mathrm{XY})$ or females $(46, \mathrm{XX})$. Test (patient) human genomic DNA was directly labelled with FITC-12-dUTP (DuPont), and control DNA was labelled with Texas Red-5dUTP (DuPont) by standard nick translation reaction. DNase I concentration was adjusted to result in an average fragment size of $500-1000 \mathrm{bp}$.

\section{HYBRIDISATION AND POST-HYBRIDISATION \\ WASHINGS}

Metaphase chromosomes from normal males were denatured for two minutes at $70^{\circ} \mathrm{C}$ in $70 \%$ formamide, $2 \times$ SSC, pH $7.0(2 \times$ SSC: 3 $\mathrm{mol} / \mathrm{l} \mathrm{NaCl}, 30 \mathrm{mmol} / 1 \mathrm{Na}_{3}$-citrate); thereafter, slides were put through an ice cold ethanol series $(70 \%, 90 \%, 100 \%)$ and air dried. Ten microlitres of hybridisation solution contained $1 \mu \mathrm{g}$ of labelled test DNA, $1 \mu \mathrm{g}$ of labelled control DNA, and $50 \mu \mathrm{g}$ of unlabelled human Cot 1 DNA (BRL Life Sciences) in 50\% formamide, $1 \times$ SSC, and $10 \%$ dextran sulphate. After denaturation at $74^{\circ} \mathrm{C}$ for seven minutes, the hybridisation mix was applied to the slide with the denatured metaphase chromosomes, covered by an $18 \times 18 \mathrm{~mm}$ coverslip, and sealed with rubber cement. After 48-72 hours' hybridisation at $37^{\circ} \mathrm{C}$, slides were washed $3 \times$ five minutes with $50 \%$ formamide and five minutes with $2 \times \mathrm{SSC}$ at $42^{\circ} \mathrm{C}$ and another five minutes with ST $(4 \times$ SSC, $0.05 \%$ Tween 20$)$ at room temperature. Slides were then dehydrated in an ethanol series $(70 \%, 90 \%, 100 \%)$ and air dried. Finally, they were counterstained with 4,6,diamino-2-phenylindole (DAPI, 0.1 $\mu \mathrm{g} / \mathrm{ml}$ ), resulting in coarse banding of the chromosomes, allowing individual chromosomes to be identified.

\section{DIGITAL IMAGE ANALYSIS}

Images for $\mathrm{CGH}$ analysis and FISH were obtained using an epifluorescence microscope (Axioplan: Zeiss, Germany) equipped with a cooled CCD camera (Photometrics) controlled by an image analysis system (Smart Capture V2.1, Digital Scientific, Cambridge, UK). For standard CGH analysis, green, red, and blue fluorescence images were captured from each high intensity, uniformly hybridised metaphase and were analysed as separate grey scale images. The image representing the blue DAPI counterstain was inverted and used for chromosome identification based on its coarse banding pattern. The mean of the individual ratio profiles of at least 10 and generally 20 metaphase spreads was calculated. The green and red fluorescence intensities were calculated and the green to red ratio profiles along the chromosome axis were displayed. For normalisation of the ratio profiles, the model value of the green to red ratio for the entire metaphase was set to 1.0. Finally, the individual ratio profiles were combined to yield the average ratio profiles, which were displayed next to the chromosome diagrams with significance intervals of 0.5 and 1.5 .
INTERPRETATION OF CGH RESULTS AND QUALITY CONTROL

Chromosomal regions with a green to red ratio above 1.5 were considered to be overrepresented (gained), whereas regions with a ratio below 0.5 were considered to be under-represented (lost). These limit values were slightly different in each experiment depending on the thresholds deduced from the analysis of negative control experiments where two sets of differently labelled normal DNA were hybridised against one another. Moreover, a telomeric area was considered to be gained when: (1) these ratios were present in both homologues of each individual metaphase as well as averaged ratio profiles in a global CGH analysis, and (2) the pattern of fluorescence intensity at the chromosomal ends for the test and control DNA was different, that is, increasing for the curve corresponding to the fluorescence intensity of test DNA while decreasing for that of control DNA. Reliability of the results was controlled in different ways. Negative and positive controls were included in each $\mathrm{CGH}$ experiment. Hybridisations of directly FITC labelled normal female DNA and Texas-Red labelled normal male DNA were used as negative controls and DNA samples with known gains and losses as positive controls. In the case of $\mathrm{JK}$, different control DNA, including parental DNA, and also different fluorochromes, were used to check the findings. FISH using appropriate telomeric or whole chromosome probes then confirmed all abnormalities found. Using this approach, no modifications in the standard $\mathrm{CGH}$ software were required.

\section{TWO COLOUR FISH}

FISH to metaphase chromosomes from patients was carried out as follows: metaphase chromosomes were denatured for two minutes at $70^{\circ} \mathrm{C}$ in $70 \%$ formamide, $2 \times \mathrm{SSC}, \mathrm{pH} 7.0$; thereafter, slides were put through an ice cold ethanol series $(70 \%, 90 \%, 100 \%)$ and air dried. In each case, appropriate biotin or digoxigenin labelled probes were first denatured and reannealed according to the manufacturers' instructions and then mixed in equal ratios. Ten microlitres of hybridisation solution containing equal amounts of each hapten labelled probe was applied to the slide with denatured chromosomes, covered by an $18 \times$ $18 \mathrm{~mm}$ coverslip, and sealed with rubber cement. After overnight hybridisation, posthybridisation washes were carried out as described above. Thereafter, slides were incubated for 10 minutes with blocking agent $(15 \%$ human serum (Sigma) in ST solution) at $37^{\circ} \mathrm{C}$. Slides were then incubated with avidin DCS conjugated to FITC (fluorscein isothiocyanate, Vector Laboratories) for 30 minutes at $37^{\circ} \mathrm{C}$ to visualise biotin labelled probes. Digoxigenin labelled probes were detected at the same time by incubation with sheep antidigoxigenin conjugated to rhodamine. No amplifications of the signals were needed. Slides were then washed 3 $x$ five minutes in ST solution at room temperature. Finally, slides were dehydrated, air dried, and mounted, as described above. 


\section{Results}

DETECTION OF CRYPTIC CHROMOSOMAL

TRANSLOCATIONS

Family 1

Patient JK was referred to the Clinical Genetics

Department because of craniofacial dysmorphism in association with tetralogy of Fallot, laryngomalacia, and inguinal hernia. He died

A
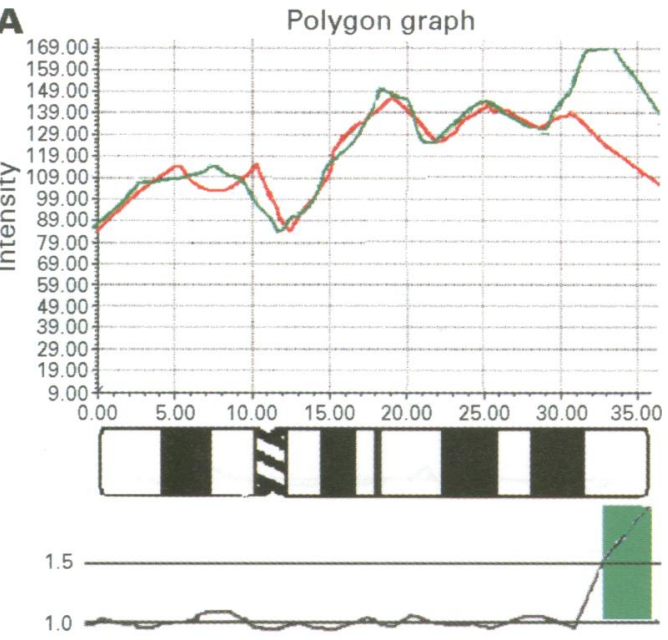

$0.5-$ CGH and targeted FISH results of $\mathcal{Y K}$ and his father using the strategy described above are depicted. $(A)$ Standard ratio profile of chromosome 17 is shown below the chromosome idiogram. The three horizontal lines below the idiogram represent the balanced state of the chromosomal copy number (middle line, ratio 1.0) and the lower (lower line) and upper (upper line) thresholds applied to detect chromosomal losses and gains, respectively. These thresholds correspond to ratios of 0.5 and 1.5 , which are the theoretical values expected for a monosomy or trisomy present in $100 \%$ of diploid cells. The polygon graph above the idiogram shows the fluorescence intensity along chromosome 17. As shown, while at the distal p arm both colours are decreasing as usual, at the distal $q$ arm the intensity of fluorescence is increasing for the green signal and decreasing for the red. This shows that the decreasing effect of fluorescence at the telomere is compensated by the extra copy of the DNA in this region. at the age of 6 months as a result of his cardiac abnormality. On taking the family history, it was noted that a paternal uncle who died in infancy from heart failure was reported to have had "classical features of Noonan syndrome". Our patient, although sharing some dysmorphic features associated with Noonan syndrome, did not have that condition but the close similarity to his uncle led to a suspicion that a cryptic translocation was segregating within the family. The results of chromosome analyses carried out on $\mathrm{JK}$, his parents, and other relatives in different service laboratories were all normal.

\section{CGH ANALYSIS USING STANDARD APPROACH}

(FIG $1 \mathrm{~A}, \mathrm{~B}$ )

CGH analysis was performed on DNA from JK. Twenty metaphases with high intensity uniform hybridisation were chosen for image analysis (see Methods). A standard type of $\mathrm{CGH}$ analysis including background correction and normalisation was performed using a fluorescence ratio (FR) threshold of 0.8-1.2. . $^{2628} 29$ Average ratio profiles showed gains for the short arms of chromosomes 21 and 14 and the distal bands of chromosomes $17 q$ and $1 p$. A loss of DNA material corresponding to the $2 \mathrm{q}$ telomeric area was also observed.

The application of a more restrictive threshold (FR: 0.5-1.5) narrowed the abnormal findings to chromosomes 17,1 , and 2 . Using the more restrictive threshold in global CGH analysis of 20 metaphases, the same results were obtained. It was also observed that although the gain in chromosome $1 p$ and deletion of $2 \mathrm{q}$ were consistent in most metaphases, they were found in only one homologue in each metaphase. On the other hand, the gain in 17q
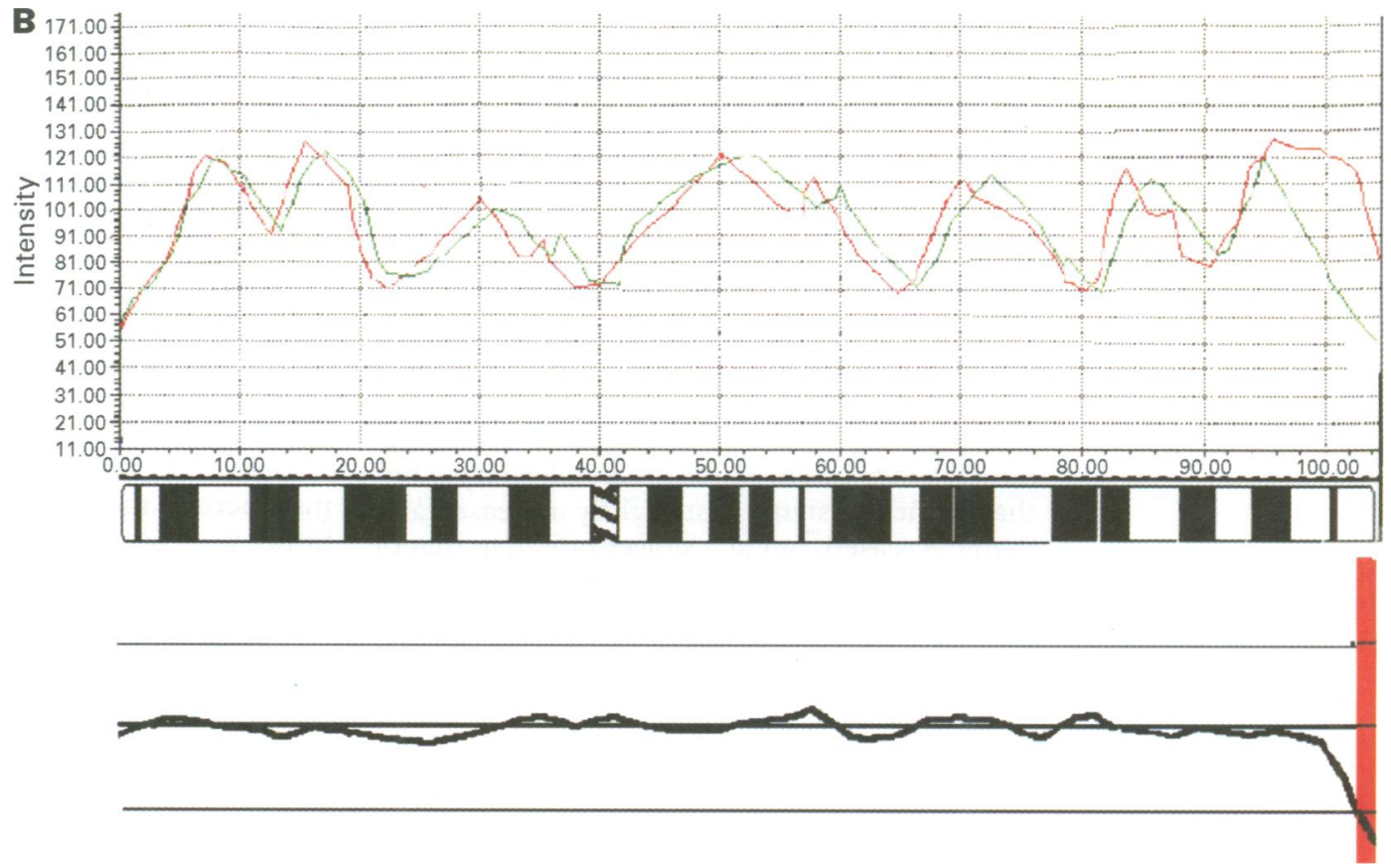

Figure 1 (B) Standard ratio profile of chromosome 2 (below the idiogram) shows a loss of DNA material related to the last band on 2q. The polygon graph (above the idiogram) shows the different gradients for fluorescence intensity secondary to the DNA copy loss at this particular area. At the p arm, the fluorescence intensities for both green and red are following the usual decreasing pattern of the chromosomal ends. 


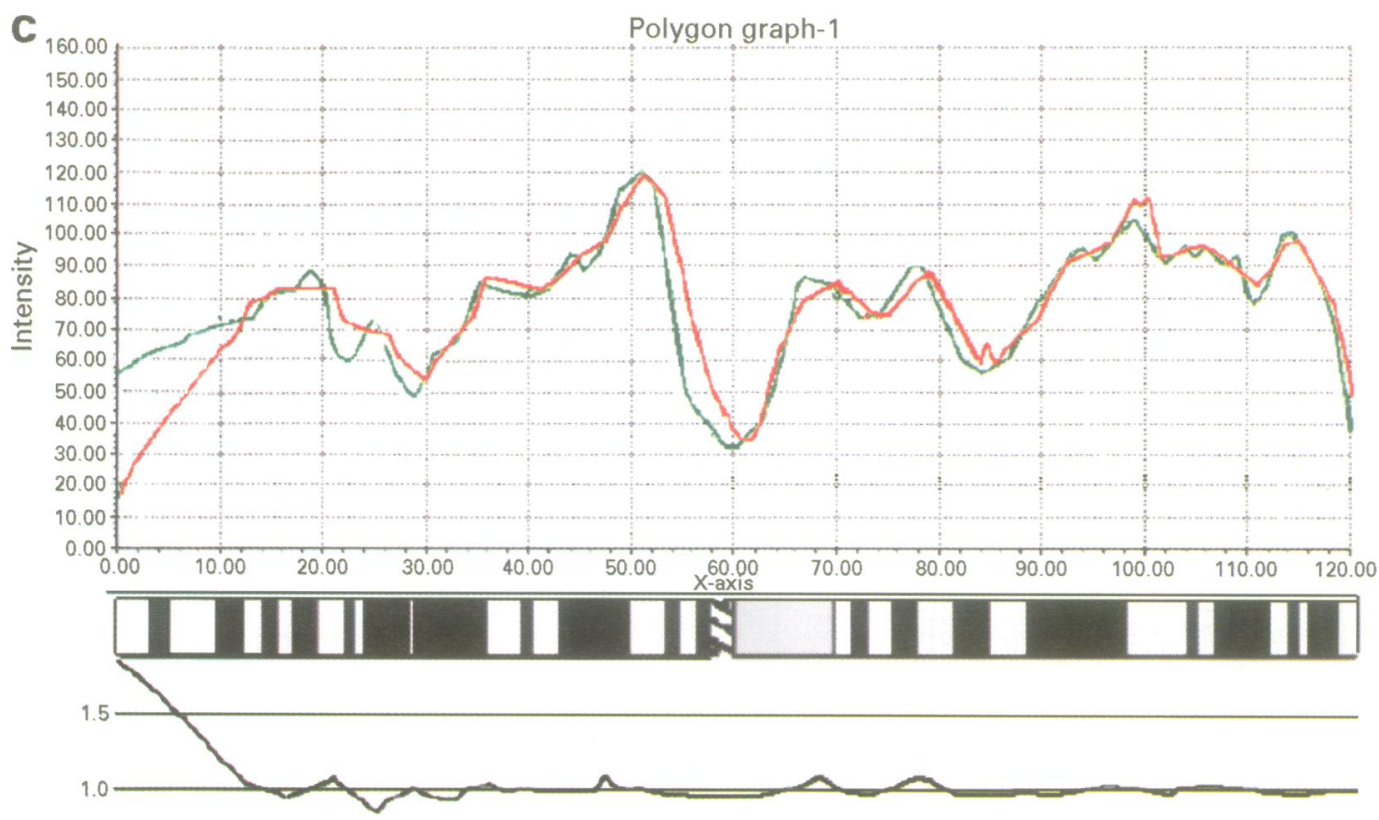

Figure 1 (C) Standard ratio profile of chromosome 1 (below the idiogram) shows a gain of DNA material related to the last band on 1p. However, fuorescence intensity quantification does not support this finding (by decreasing for both colours).

was consistently present in both homologues of most metaphases.

In assessing the diagnostic significance of the above observations, we took into account that we had failed to observe similar findings in the telomeric areas examined in $2000 \mathrm{CGH}$ images from 100 healthy subjects, nor were these telomeric changes observed in a larger series of approximately $48000 \mathrm{CGH}$ images from 200 subjects with different abnormalities not involving the telomeric regions.

CGH ANALYSIS BASED ON THE PATTERN OF FLUORESCENCE INTENSITY AT CHROMOSOME ENDS (FIG $1 \mathrm{~A}, \mathrm{~B}, \mathrm{C}$ )

To investigate the significance of the results obtained by standard CGH analysis, the fluorescence intensity of red and green signals corresponding to test and control DNA in the 20 normalised CGH images was measured. Fluorescence intensity of the green signal (patient DNA) at both homologues of the 17q telomere, both before and after averaging, increased while that of the red signal corresponding to control DNA decreased. At distal $2 \mathrm{q}$, however, both before and after averaging, fluorescence intensity for green fell sharply but that of the red signal first slightly increased and then decreased, but at a slower rate than that of the green signal. Quantification of the fluorescence intensities for both red and green at the $1 \mathrm{p}$ region showed decreasing patterns despite the ratio findings.

To rule out sources of error, the CGH experiment was repeated with the fluorochromes reversed (red for test and green for control DNA). Various different control samples were used, including DNA from both parents and several unrelated subjects. Under all these conditions the findings for chromosomes 17 and 2 remained consistent. These results suggested strongly that there was at least one extra copy of terminal chromosome 17q telomeric region, and if the patient had an unbalanced translocation, the extra material was most likely translocated to the distal end of $2 q$, leading to partial monosomy/trisomy for chromosomes $2 \mathrm{q}$ and $17 \mathrm{q}$.

As expected for chromosomally balanced karyotypes, CGH analysis showed no gain or loss of DNA material in any relative connecting the patient and his dead uncle, nor were there any CGH abnormalities in other healthy family members.

CONFIRMATION OF CGH RESULTS BY FISH

To confirm the $\mathrm{CGH}$ findings, FISH was carried out on metaphase chromosomes from JK using commercial probes specific for the telomeric regions of chromosomes $17 q$ (Oncor) and $2 q(\mathrm{LI})$. Three signals for $17 \mathrm{q}$ telomere were detected, two located at $17 \mathrm{q}$, and the third signal was translocated to a $2 \mathrm{q}$ telomere (fig 1D). Conversely, the chromosome $2 \mathrm{q}$ telomeric probe showed only one signal that was normally located on one homologue of chromosome 2 (fig 1D).

Using the same FISH probes on the other members of the family, JK's father and grandfather were found to be carriers of the balanced translocation (fig 1E), while the mother and grandmother had normal results.

The size of the abnormality deduced from the averaged relative length of each chromosome to total haploid autosome was estimated to $b e \sim 10 \mathrm{Mb}$ for the duplication of $17 \mathrm{q}$ and $\sim 4$ $\mathrm{Mb}$ for the $2 \mathrm{q}$ deletion (fig $1 \mathrm{~A}, \mathrm{~B}$ ).

\section{Family 2}

Patient CL, a 32 month old girl, was referred to the Clinical Genetics Department with mild gross motor and speech delay, a small ventricu- 


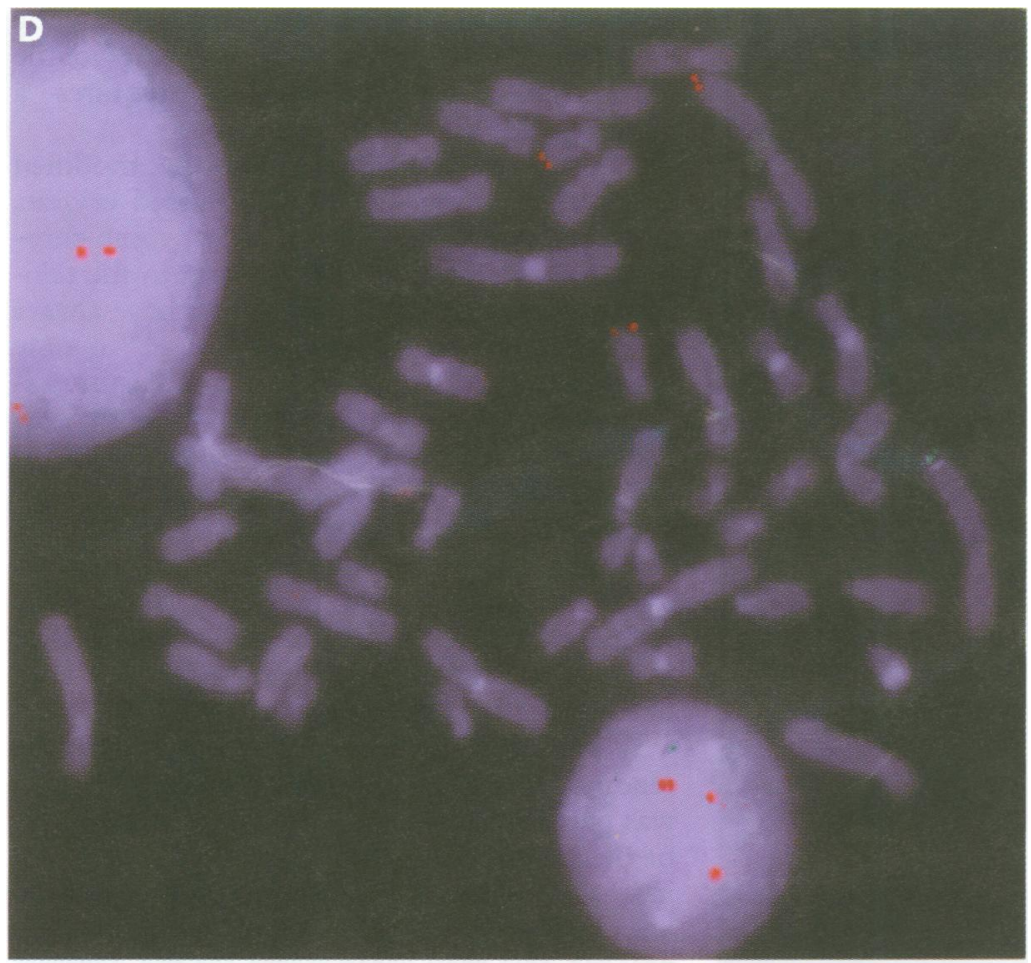

E

Figure 1 (D) Two colour FISH to metaphase from $¥ K$ using chromosomes $2 q$ and $17 q$ telomeric probes: red signals correspond to the chromosome 17 and green signal corresponds to the chromosome 2 telomeric probe. The picture shows an unbalanced $2 q ; 17 q$ translocation resulting in, respectively, partial monosomy/trisomy for these chromosomes. (E) FISH to metaphase from $\mathcal{Y K}$ 's father using the same probes, showing a balanced $2 q ; 17 q$ translocation.

lar septal defect, and minor facial dysmorphism, including low set ears, a flat philtrum, and a wide mouth. In addition, she had bilateral vesicoureteric reflux (VUR). In the family history, a male first cousin had identical, mild developmental delay, minor facial dysmorphism, and had undergone surgery for VUR. Both cousins and their parents had normal $G$ banded karyotypes (400-500 band level), but the presence of virtually identical phenotypes in male and female cousins who are related through healthy parents again led to the suspicion that a cryptic chromosome translocation was segregating in this family.

DNA from CL was subjected to $\mathrm{CGH}$ analysis using our modified technique and this suggested gain of $20 p$ telomere and loss of $4 q$ telomere. The size of the abnormality was estimated to be $7 \mathrm{Mb}$ for the $20 \mathrm{p}$ duplication and $10 \mathrm{Mb}$ for the $4 \mathrm{q}$ deletion. The results of FISH analysis using the specific telomeric probes for these chromosome regions (20p (Oncor) $4 q$ (LI)) confirmed the $\mathrm{CGH}$ results, identifying an unbalanced translocation between the telomeric bands of chromosomes $4 q$ and $20 p$ (fig 2A). Both affected cousins had partial trisomy for chromosome 20p and partial monosomy for chromosome 4q. In turn, the mother of one affected cousin and the father of the other were found to carry a balanced reciprocal $4 q ; 20 p$ translocation which was inherited from their own mother (fig 2B).

\section{Family 3}

Patient SB, a first born male infant, was referred to the Clinical Genetics Department at the age of 2 years when moderate mental handicap was diagnosed. He had no major congenital abnormalities and minimal dysmorphism but in the family history his maternal aunt, FM, had severe, unexplained mental handicap. This 32 year old woman was examined and found to have short stature (height $140 \mathrm{~cm}$ ), microcephaly (OFC $51 \mathrm{~cm}$ ), and non-specific facial dysmorphism. In view of this family history, high resolution chromosome analyses were repeated on both patients and their relatives on several occasions and, although no abnormality was detected, an underlying cryptic chromosome translocation was always suspected on clinical grounds.

Modified CGH was performed on a DNA sample from FM and showed partial gain and loss of DNA material corresponding to the distal bands of chromosomes $20 \mathrm{q}$ and $10 \mathrm{q}$, respectively. The size of the duplicated $20 \mathrm{q}$ was estimated to be $5 \mathrm{Mb}$ and loss of $10 \mathrm{q}$ region was about $10 \mathrm{Mb}$. FISH studies using $10 \mathrm{q}$ and 20q telomeric probes (LI) confirmed partial trisomy for $20 \mathrm{q}$ and partial monosomy for $10 \mathrm{q}$. The same unbalanced abnormality was also detected in SB (fig 3A, B). The balanced reciprocal translocation was detected by FISH in SB's mother (fig 3C, D) and maternal grandfather, but it was not present in a maternal aunt of SB.

\section{Discussion}

A cryptic translocation is one which cannot be observed by conventional cytogenetic analysis, either because the size and banding pattern of the chromosome regions involved are too similar for the exchange to be detected, or because the size of the exchanged segments is close to the limit of resolution of the cytogenetic technique. ${ }^{1-4}$ Small translocations involving telomeric regions of chromosomes are especially difficult to detect since most telomeres have a similar banding pattern. To date, detec- 


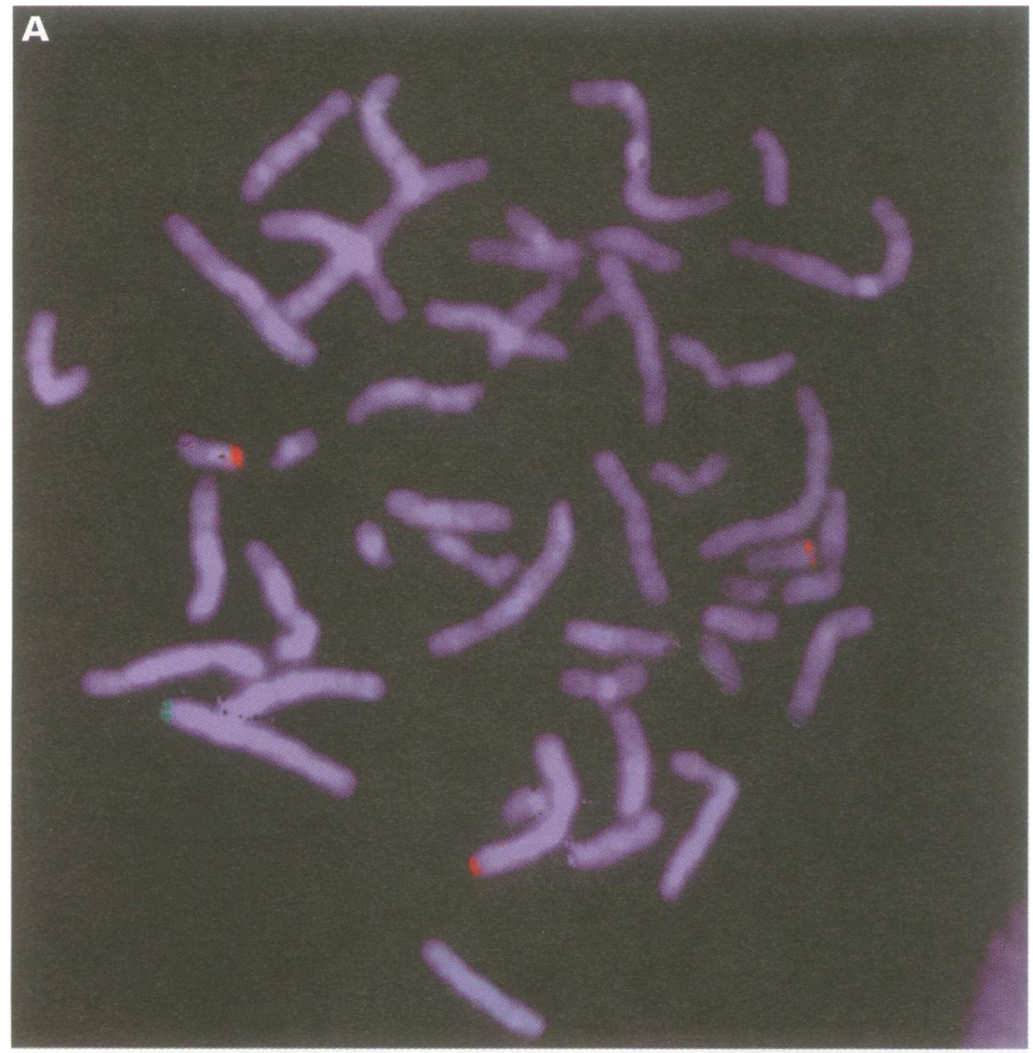

B

Figure 2 (A) FISH to metaphase of CL using telomere probe for 20p and $4 q$ showing the presence of three red signals corresponding to three copies of 20p telomere region and one green signal corresponding to $4 q$ telomere. (B) Metaphase of CL's balanced carrier father using the same probes. studies is also crucial. To diagnose cryptic translocations without a specific clue to the chromosome segment involved requires a genome wide screening technique.

The data presented here show that modified CGH analysis can detect unbalanced cryptic, telomeric translocations of at least some chromosomes. Previously, data relating to the telomeric regions of the chromosomes have always been excluded from CGH analysis, ${ }^{25}{ }^{26}$ mainly because the green and red fluorescence intensities gradually decrease at the chromosome telomeres, and therefore a reliable colour ratio is not possible with the currently used algorithms. However, by introducing a new strategy involving close attention to previously ignored telomeric regions and the application of a highly restrictive threshold, we have been able to show translocations involving the telomeres of $2 \mathrm{q}$ and $17 \mathrm{q}, 20 \mathrm{p}$ and $4 \mathrm{q}, 20 \mathrm{q}$ and $10 \mathrm{q}$.

It was noted that partial trisomy was more readily seen by the modified technique than was partial monosomy. This phenomenon may be because of the behaviour of the fluorescence intensities at the chromosome terminals: when an extra copy of DNA from a telomere is present, this may compensate for the decreasing fluorescence intensity at the chromosome end and the signal shows a sharp rise while, in contrast, the intensity of signal corresponding to control DNA gradually decreases at chromosome ends. When this pattern is present and the above criteria are adhered to, diagnosis of DNA gain corresponding to at least one extra telomere is possible. Conversely, in partial monosomies with loss of one copy of the terminal band, there is less difference in the patterns of fluorescence intensity in control and test DNA. While the intensity of the test signal decreases sharply, the fluorescence intensity of the control signal increases slightly at first and then gradually decreases. The initial slight increase might be explained by increased hybridisation of control DNA when it competes with less test DNA from the deleted chromosome segment. This difference in the gradient of the fluorescence intensities, although less obvious than that associated with the partial trisomies, was sufficient to lead to the detection of DNA deletion in the three families described here.

There is no reported evidence that the detection of partial monosomies using CGH has proved difficult, at least with respect to larger sizes of DNA loss. On the other hand, DNA losses of the size seen here, especially those involving telomeric areas, have only rarely been described using other techniques and a study of the sensitivity of CGH in this situation is warranted. We believe that our results to date justify using the modified CGH technique to screen a larger series of patients where clinical genetic evidence points to cryptic rearrangement.

Although findings related to the telomeric areas of different chromosomes have previously been regarded as unreliable, when the standard CGH approach detects gain or loss of DNA material of one particular telomere, this observation should not be discounted. Thus, we can cytogenetic clues that point to a specific chromosome deletion or duplication syndrome. The availability of DNA probes that permit informative DNA or molecular cytogenetic 

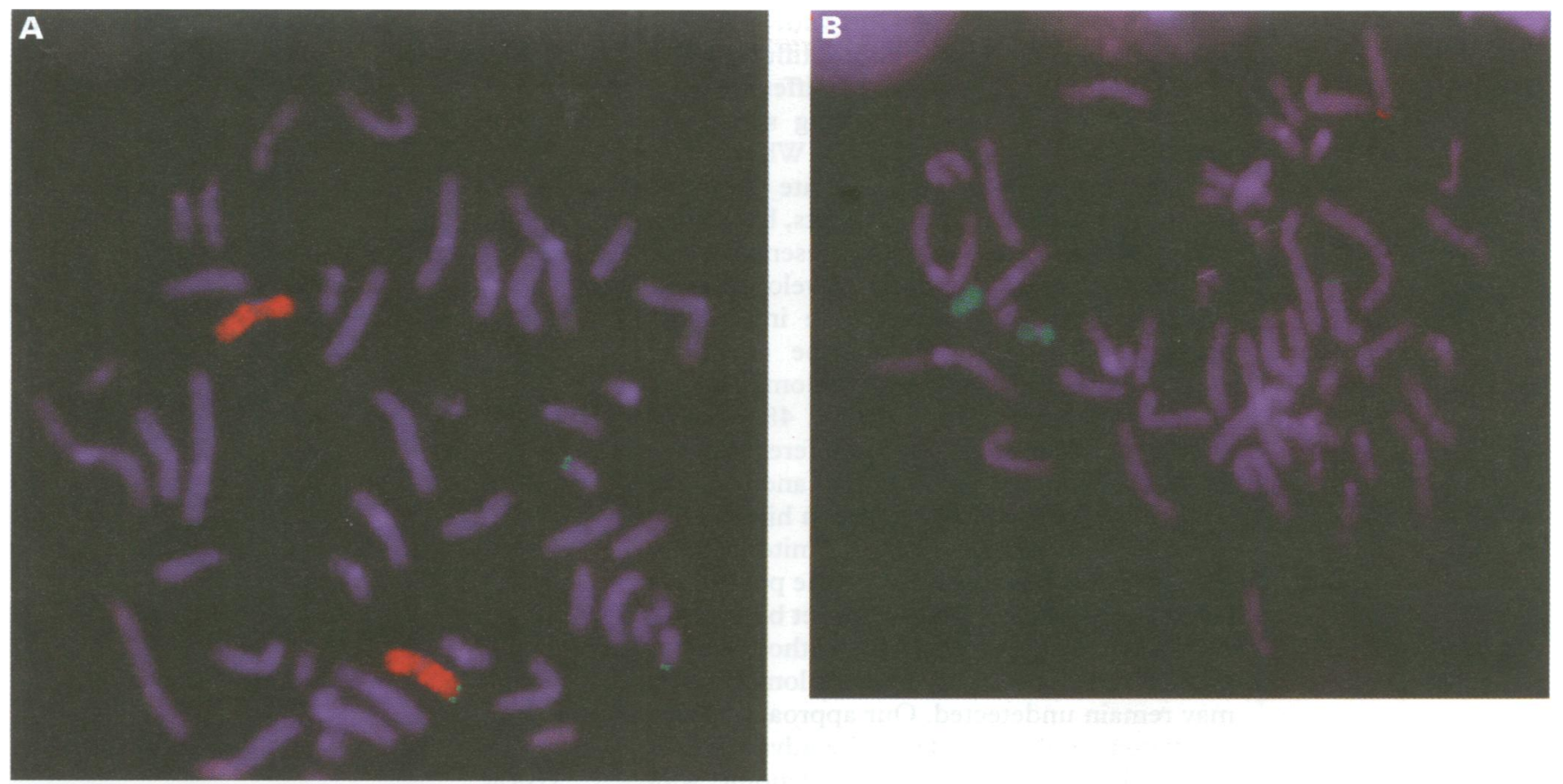

C

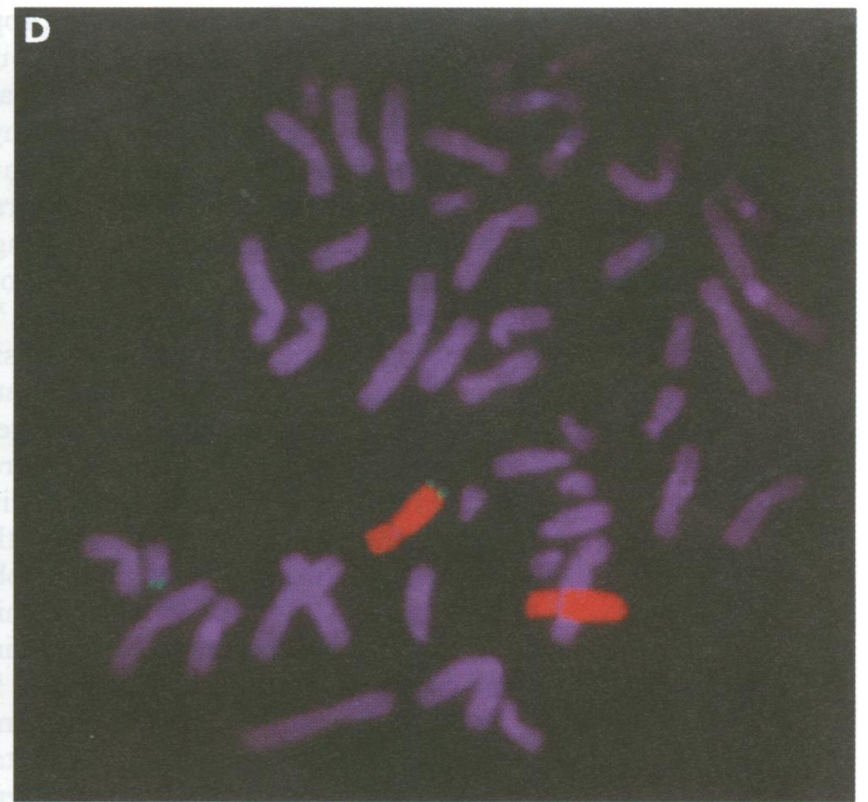

Figure 3 FISH to metaphase of SB using whole chromosome libraries for chromosome 10 (red) and 20 (green) and telomere probes for $20 \mathrm{q}$ (green) and $10 q$ (red), showing the presence of three green signals corresponding to three copies of $20 q$ telomere region $(A)$ and one red signal corresponding to $10 q$ telomere (B). Metaphase of $S B$ 's balanced carrier mother using the same probes $(C, D)$.

summarise the strategy that we suggest for $\mathrm{CGH}$ analysis of telomeric areas as follows. (1) In cases of gain of DNA material: using a restrictive threshold, global $\mathrm{CGH}$ analysis should show similar findings in both homologues in most metaphases; intensity of fluorescence corresponding to test DNA at a telomeric area should increase, while fluorescence intensity of control DNA decreases. (2) When loss of DNA material is suggested, the intensity of fluorescence corresponding to the control DNA increases slightly at first and then decreases, but fluorescence intensity of test DNA decreases sharply from the beginning of the deleted segment. These patterns must be present in the averaged profile as well as in most individual chromosome profiles. Targeted
FISH studies using appropriate probes are then used to confirm the CGH results.

Since the technique depends on analysis of patients' DNA, it is not dependent on a high quality chromosome preparation. Although balanced translocations cannot be detected by this technique, this is only a major disadvantage if there are no living affected subjects. Stored DNA from subjects who have died may also be studied, emphasising the importance of storing a DNA sample from people with unexplained mental handicap. Targeted FISH studies will detect carriers of the balanced abnormality that led to the specified chromosome imbalance in the proband, or confirm de novo mutation in the proband if both parents have normal FISH results. 
Another approach to genome wide analysis is provided by combinatorial multifluor FISH $(\mathrm{M}-\mathrm{FISH})^{30}{ }^{31}$ where up to 27 different chromosome paint probes (including some arm specific paints) have been used. Whole chromosome FISH probes can facilitate the detection of some small rearrangements, but telomeric regions are often under-represented in the painting probes. ${ }^{32}$ Recently developed telomeric probes $^{33}$ may prove more informative using M-FISH or a multiprobe coverslip ${ }^{34}$ device. However, to cover the telomeric areas of all chromosomes in M-FISH, 48 different telomeric probes in at least 24 different colours (ideally 48 colours) are required ${ }^{3}$ and the multiprobe coverslip device requires a high quality chromosome preparation of high mitotic index. Although the device has shown the presence of known abnormalities, it has not yet been tested in a blind study. With both methods, subtle cryptic deletions outside the telomeric area may remain undetected. Our approach, if substantiated, could provide all the advantages of the $\mathrm{CGH}$ technique including genome wide screening for unbalanced abnormalities and dependence on DNA rather than metaphase spreads. The $\mathrm{CGH}$ analysis took approximately one working day following 48 hours' incubation for hybridisation. FISH analysis for confirmation involved overnight hybridisation and a half day for detection and analysis.

The occurrence of telomeric translocations has been explained by the finding that nonhomologous chromosomes share common telomere associated repeat sequences. ${ }^{13}$ These may result in mispairing during meiosis and thus facilitate translocation. The fact that the highest gene concentrations in the human genome are in telomeric bands of metaphase chromosomes ${ }^{22}$ makes the study of these abnormalities particularly important for understanding the causes of mental handicap, a common problem which affects up to $3 \%$ of all school age children and is of idiopathic or unexplained origin in up to $80 \%$ of cases. ${ }^{35}{ }^{36}$ Recently, Flint $e t a l^{5}$ found three cases of cryptic de novo rearrangements among 99 patients with idiopathic mental handicap who were screened for subtelomeric chromosomal abnormalities with a combination of molecular cytogenetic and DNA techniques. All three cases diagnosed in that report had de novo rearrangements whereas the patients described here were selected for detailed study because inheritance patterns and clinical features strongly suggested they suffered familial chromosomal imbalance. Our patients also had larger, cryptic rearrangements (in the range 4-10 $\mathrm{Mb}$, see Results and fig 1A, B) which were only detected after we adapted $\mathrm{CGH}$ to work successfully at its highest reported resolution in clinical cytogenetics, close to its ultimate theoretical resolution using the current available technology. Although CGH analysis is presently unable to detect abnormalities less than $4 \mathrm{Mb}$ in size, given our discovery of the important contribution of larger cryptic rearrangements to familial, idiopathic mental handicap, there is a high priority to investigate the use of $\mathrm{CGH}$ as a tool for genome wide screening in a larger series of sporadically affected subjects with idiopathic mental handicap.

We thank Dr Julie McGaughran (Manchester) for supplying clinical details and blood samples from relatives in family 1 , and we also thank colleagues in the cytogenetics laboratories at the Duncan Guthrie Institute for preparing and examining many conventional cytogenetic preparations from patients and their families. SRG received a scholarship from the Ministry of Health and Medical Education, Iran.

1 Lamb J, Harris PC, Lindenbaum RH, et al. Detection of breakpoints in submicroscopic chromosomal translocation, illustrating an important mechanism for genetic disease. Lancet 1989;ii:819-24.

2 Overhauser J, Bengtsson U, McMahon J, et al. Prenatal diagnosis and carrier detection of a cryptic translocation by using DNA markers from the short arm of chromosome 5. Am f Hum Genet 1989;45:296-303.

3 Ledbetter DH. Minireview. Cryptic translocations and telomere integrity. Am $\mathcal{F}$ Hum Genet 1992;5:451-6.

4 Wilkie AOM. Detection of cryptic chromosomal abnormalities in unexplained mental retardation: a general strategy using hypervariable subtelomeric DNA polymorphisms. Am Ұ Hum Genet 1993;53:688-701.

5 Flint J, Wilkie AOM, Buckle VJ, Winter RM, Holland AJ, McDermid HE. The detection of subtelomeric chromosomal rearrangements in idiopathic mental retardation. Nat Genet 1995;9:132-9.

6 Wilkie AOM, Buckle VJ, Harris PC, et al. Clinical features and molecular analysis of the alpha thalassemia/mental retardation syndromes. I. Cases due to deletions involving retardation syndromes. I. Cases due to deletions involving chromosom

7 Kuwano A, Ledbetter SA, Dobyns WB, Emanuel BS, Ledbetter DH. Detection of deletions and cryptic translocations in Miller-Dieker syndrome by in situ hybridisation. Am F Hum Genet 1991;49:707-14.

8 Park VM, Gustashaw KM, Wathen TM. The presence of interstitial telomeric sequences in constitutional chromosome abnormalities. Am $\mathcal{f H u m}$ Genet 1992;50:914-23.

9 Alvarado M, Bass HN, Caldwell S, Jamehdor M, Miller AA, Jacob P. Miller-Dieker syndrome: detection of a cryptic chromosome translocation using in situ hybridisation in a family with multiple affected offspring. Am $尹$ Dis Child 1993;147:1291-4

10 Migeon BR, McGinniss MJ, Antonarakis SE, et al. Severe haemophilia A in a female by cryptic translocation: order and orientation of factor VIII within Xq28. Genomics 1993; and orien

11 Reeve A, Norman A, Sinclair P, et al. True telomeric translocation in a baby with the Prader-Willi phenotype. $A m \mathcal{F}$ Med Genet 1993;47:1-6.

12 Kohler A, Hain J, Muller U. Familial half cryptic translocation $\mathrm{t}(9 ; 17)$. $\mathcal{F}$ Med Genet 1994;31:712-14.

3 Masuno M, Imaizumi K, Nakamura M, Matsui K, Goto A, Kuroki Y. Miller-Dieker syndrome due to maternal cryptic translocation $\mathrm{t}(10 ; 17)(\mathrm{q} 26.3 ; \mathrm{p} 13.3)$. Am $\mathcal{F}$ Med Genet 1995;59:441-3.

14 Estop AM, Mowery Rushton PA, Cieply KM, et al. Identification of an unbalanced cryptic translocation $t(9$; 17)(q34.3;p13.3) in a child with dysmorphic features. $\mathcal{f}$ Med Genet 1995;32:819-22.

15 Wintle RF, Costa T, Haslam RHA, Teshima IE, Cox DW Molecular analysis redefines three human chromosome 14 Molecular analysis redefines three human
deletions. Hum Genet 1995;95:495-500.

16 Scott JA, Wenger SL, Steele MW, Chakravarti A. Down syndrome consequent to a cryptic maternal 12p;21q chromosome translocation. Am f Med Genet 1995;56:67-71.

17 Blennow E, Bui TH, Wallin A, Kogner P. Monosomy 1p36.31-33>pter due to a paternal reciprocal ranslocation: prognostic significance of FISH analysis. $\mathrm{Am}$ f Med Genet 1996;65:60-7.

18 Burke LW, Wiley JE, Glenn CC, et al. Familial cryptic translocation resulting in Angelman syndrome: implications for imprinting or location of the Angelman gene? Am $7 \mathrm{Hum}$ Genet 1996;58:777-84.

19 Reid E, Morrison N, Barron L, et al. Familial WolfHirschhorn syndrome resulting from a cryptic translocation: a clinical and molecular study. $\mathcal{f}$ Med Genet 1996;33:197-202.

20 Smith DP, Floyd M, Say B. Detection of a familial cryptic translocation by fluorescent in situ hybridisation. $\mathcal{F}$ Med Genet 1996;33:84.

21 Van Zelderen Bhola SL, Breslau Siderius EJ, Beverstock GC, et al. Prenatal and postnatal investigation of a case with Miller-Dieker syndrome due to a familial cryptic translocation $t(17 ; 20)$ (p13.3; 113.3 ) detected by fluorescence in situ hybridization. Prenat Diagn 1997;17:173-9.

22 Saccone S, De Sario A, Dellae-Valle G, Bernardi G. The highest gene concentrations in the human genome are in the telomeric bands of metaphase chromosomes. Proc Natl Acad Sci USA 1992;89:4913-17.

23 Kallioniemi A, Kallioniemi OP, Sudar D, et al. Comparative genomic hybridization for molecular cytogenetic analysis of solid tumors. Science 1992;258:818-21.

24 Du Manoir S, Speicher MR, Joos S, et al. Detection of complete and partial chromosome gains and losses by comparative genomic in situ hybridization. Hum Genet 1993;90:590-610.

25 Kallioniemi OP, Kallioniemi A, Piper J, et al. Optimizing comparative genomic hybridization for analysis of DNA 
sequence copy number changes in solid tumors. Genes Chrom Cancer 1994;10:231-43.

26 Bryndorf T, Kirchhoff M, Rose H, et al. Comparative genomic hybridization in clinical cytogenetics. $\mathrm{Am}$ f Hum Genet 1995;57:1211-20.

27 Erdel M, Duba HC, Verdorfer I, et al. Comparative genomic hybridization reveals a partial de novo trisomy $6 \mathrm{q} 23$-qter in an infant with congenital malformations: delineation of the phenotype. Hum Genet 1997;99:596-601.

28 Lundsteen C, Maahr J, Christensen B, et al. Image analysis in comparative genomic hybridization. Cytometry 1995;19: in comp.

29 Piper J, Rutovitz D, Sudar D, et al. Computer image analysis of comparative genomic hybridization. Cytometry 1995;19. 10-26.

30 Speicher MR, Ballard DC, Ward DC. Karyotyping human chromosomes by combinatorial multi-fluor FISH. Nat Genet 1996;12:368-75.

31 Schrock E, Du Manoir S, Veldman T, et al. Multicolor spectral karyotyping of human chromosomes. Science 1996;273: 494-7.
32 Rosenberg C, Blakemore KJ, Kearns WG, et al. Analysis of reciprocal translocations by chromosome painting: applications and limitations of the technique. Am f Hum Genet 1992;50:700-5.

33 National Institutes of Health and Institute of Molecular Medicine Collaboration. A complete set of human Collaber telomeric probes and their clinical application. Nat Genet
1996;14:86-9.

34 Knight SJL, Horsley SW, Regan R, et al. Development and clinical application of an innovative fluorescence in situ hybridization technique which detects submicroscopic hybridization technique which detects submicroscopic rearrangem

35 Roeleveld N, Zielhus GA. The prevalence of mental retardation: a critical view of recent literature. Dev Med Child Neurol 1997;39:125-32.

36 Yeargin-Allosop M, Murphy CC, Cordero JF, Decoufle P, Hollowell JG. Reported biomedical causes and associated medical conditions for mental retardation among 10-yearold children, metropolitan Atlanta, 1985 to 1987. Dev Med Child Neurol 1997;39:142-9. 